\title{
The ground dominance effect in the perception of 3-D layout
}

\author{
ZHENG BIAN and MYRON L. BRAUNSTEIN \\ University of California, Irvine, California \\ and \\ GEORGE J. ANDERSEN \\ University of California, Riverside, California
}

\begin{abstract}
The relative effectiveness of the ground surface and other environmental surfaces (the ceiling and sidewalls) in determining perceived layout was investigated in five experiments and a real-world demonstration. In the first three experiments, two vertical or horizontal posts were positioned between two surfaces (ground and ceiling in all three experiments, left wall and right wall in Experiment 1), and optical contact was manipulated so that the two surfaces provided contradictory information about the relative distances of the posts. Observers judged which of the two posts appeared to be closer. In Experiment 4 , to control the height on the posts at which the distance judgments were made, a blue dot was attached to both vertical posts at varying heights and observers judged which dot appeared closer. In Experiment 5, the posts were replaced by two gray ellipses to eliminate the effects of the regular shape and texture. Our findings were that (1)among all four surfaces tested, observers showed a preference to respond according to the optical contact information provided by the ground surface-a ground dominance effect, (2) this effect did not depend on the height of the posts in the image, (3) as the scene was tilted away from a ground/ceiling orientation, the ground dominance effect decreased, and (4) this effect was not due to the location of the judgment.
\end{abstract}

The perception of 3-D scenes usually involves an integration of information about overall scene depth, the layout or relative position of objects in the scene, and the properties of the objects within the scene (Andersen, Braunstein, \& Saidpour, 1998). The information may be either viewer centered, providing absolute or relative distances from the observer to the locations in the scene, or object centered, providing distances among locations in the scene. There is a considerable body of research focusing on the effectiveness of different sources of information used in the perception of individual objects and surfaces in 3-D scenes, such as texture (e.g., Andersen et al., 1998; Stevens, 1981; Todd \& Akerstrom, 1987), motion parallax (e.g., Braunstein \& Andersen, 1981; Rogers \& Graham, 1979), and binocular disparity (e.g., Gillam, Flagg, \& Finlay, 1984; Norman \& Todd, 1998). Another stream of research has studied the integration of different cues in indicating depth, such as motion and disparity (e.g., Turner, Braunstein, \& Andersen, 1997), motion and

This research was supported by National Institutes of Health Grant EY-12437. Portions of this research were presented at the 2003 meeting of the Psychonomic Society, Vancouver, BC, and the 2004 meeting of the Vision Sciences Society, Sarasota, Florida. We thank Allison Sekuler, H. A. Sedgwick, and an anonymous reviewer for helpful suggestions and Cary Feria, Rui Ni, and Huiying Zhong for helpful discussions. Correspondence should be addressed to M. L. Braunstein, 3151 Social Science Plaza, University of California, Irvine, CA 92697-5100 (e-mail: mlbrauns@uci.edu). texture (e.g., Braunstein, 1968), and disparity and shading (Tittle, Norman, Perotti, \& Phillips, 1998). These studies have contributed to an understanding of different sources of information for the perception of objects and surfaces and how these sources of information interact, but relatively little is known about how information about scene depth, layout, and object properties from viewer-centered and object-centered sources is integrated to provide a perception of a 3-D scene. In this article, we will consider the role of the ground surface in providing the layout of objects in a 3-D scene.

Alhazen (1989) proposed that the continuous ground surface between objects is important in judging their relative distances. Gibson (1946, 1950; see also Sedgwick, 1983) emphasized the central role of a continuous surface in 3-D perception: "The problem of three-dimensional vision, or distance perception, is basically a problem of the perception of a continuous surface which is seen to extend away from the observer .... An array of objects by themselves does not make up visual space.... The visual world consists of object-surfaces on a background of an extended ground surface" (Gibson, 1946, p.420). Gibson developed his ideas into a ground theory, which he defined as "the possibility that there is literally no such thing as a perception of space without the perception of a continuous background surface" (Gibson, 1950, p. 6).

If a continuous background surface underlies the perception of the layout of a 3-D scene, objects must be associated with positions along this surface. Gibson (1950) 
proposed that the perceived distance of an object can be determined by the "optical contact" between the object and the background surface. Optical contact refers to the contact between the image of an object and the image of a background surface in a 2-D projection, as opposed to physical contact between the object and the background in the 3-D scene. In a demonstration of the effectiveness of optical contact as information for the perception of relative distance, Gibson (1950) created a scene with two objects and a background surface (see Figure 1.) One object was lifted above the surface by a concealed support, so that its optical contact position indicated a distance greater than its actual position. The object with the more distant optical contact position appeared more distant, even though it was actually closer.

Several recent studies have confirmed the importance of the ground plane in depth perception. Meng and Sedgwick $(2001,2002)$ proposed that surfaces, especially the ground plane, provide a common reference frame for both egocentric distance (distance from an object to the observer) and exocentric distance (distance between two objects in the scene) and therefore are the basis for the perception of 3-D layout. The location of an object is first specified by its local contact with an adjacent surface. This information can propagate to the ground plane and other environmental surfaces through "nested" contact relations, which are hierarchical and may have many levels. Meng and Sedgwick (2001) used computer-generated displays to ask observers to adjust the position of an arrow on a track lying on the ground to match the distance of a cube lying on a platform, which in turn was lying on or suspended above the ground plane. They found that, without varying the actual position of the cube, the perceived location of the cube could be mediated by varying the height of the platform relative to the ground plane and consequently the optical contact between the platform and the ground plane. This showed that the perceived distance could be efficiently integrated through nested contact relations among surfaces. In Meng and Sedgwick's (2002) study, they placed the track on another platform and varied (1) the relative height of the platform supporting the cube and that supporting the track, and (2) the relative depth of the front surface of the two platforms. They found that the judgments were more accurate when the two platforms had the same height or when the depth of the front surfaces was the same, demonstrating that local information was not integrated completely with global information as the nested contact relations became more complicated.

Since Gibson (1950) emphasized the importance of a "continuous" surface in his ground theory, Sinai, Ooi, and He (1998) examined the effect of a texture discontinuity on the ground plane in an outdoor environment. They found that perceived distance, measured either by perceptual judgments of absolute distance or visually cued blind walking, was accurate on a continuous ground surface, underestimated when the ground surface was composed of two distinct textured regions, and overestimated when the ground surface was interrupted by a gap. Feria, Braunstein, and Andersen (2003) used a computergenerated display to examine this "discontinuity effect" and found that it exists even when the same texture is present in both regions, but one region is offset horizontally to produce an implicit contour.

These studies provided information about the important role of the ground surface in perceiving the visual world, but they did not address the question of whether other environmental surfaces, such as the ceiling and walls, are as effective as the ground surface in mediating the perception of layout in 3-D scenes. Gibson (1950, 1979) believed that the ground surface was of special importance among environmental surfaces for several reasons. First, human beings, as well as most land-dwelling animals, are "terrestrial" and almost always depend on the ground plane for locomotion. Second, the ground plane is universal and exists in all spaces in which we can live. Ceilings and walls, on the other hand, are usually artificial surfaces and are not universal.

Several recent studies, mostly by He and Nakayama (1992, 1994a, 1994b, 1995), have confirmed the importance of a continuous surface in mediating higher level visual functions, including visual search (He \& Nakayama, 1992, 1995), motion perception (He \& Nakayama, 1994a), and visual texture segregation (He \& Nakayama, 1994b). They proposed that the information picked up from the retinal image is not directly linked to higher level visual processing but is instead connected by a stage that they refer to as "surface representation." This surface representation stage maximizes the economy of representation, allowing us to operate efficiently in the visual environment. Through evolution and learning, our perceptual system should have learned to encode the most significant, most general parts of our visual environment, which are the background surfaces, and to provide layout in-
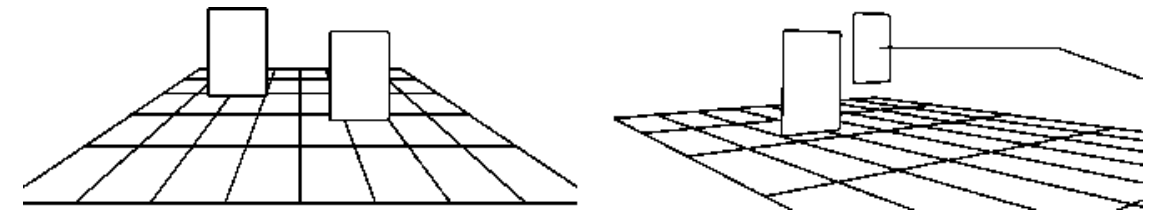

Figure 1. Schematic of Gibson's (1950, p. 179) demonstration showing a front view (left panel) and back view (right panel). The card on the left in the front view is suspended above the surface. 
formation about scenes accordingly (Nakayama, He, \& Shimojo, 1995). One prediction of this view is that the ground surface, being universal in visual space, has a perceptual advantage over all other environmental surfaces. This was supported by McCarley and He (2000), who used a visual search task to compare the effectiveness of a ground-like surface and a ceiling-like surface in mediating visual attention. Observers were found to respond faster when the target was on a ground-like surface than when it was on a ceiling-like surface.

All these studies suggest an advantage of the ground surface over other environmental surfaces in organizing 3-D scenes, but the effectiveness of different environmental surfaces in determining perceived layout in 3-D scenes has not been previously investigated. The present study compared the relative effectiveness of the ground surface and other environmental surfaces, especially the ceiling, in determining the perceived layout of 3-D scenes. We presented observers with a scene in which the ground surface and the ceiling, or two sidewalls, were present at the same time and provided contradictory layout information. This was achieved by manipulating the optical contact of two posts with the ground surface and the ceiling, or with two sidewalls. The question we were asking was, when the two surfaces provide contradictory information, which surface will observers use to determine the layout of the scene?

Five experiments and one demonstration were conducted. The first experiment compared judged layout for objects in contact with the ground or ceiling and objects in contact with a right or left wall. The second experiment examined ground and ceiling contact with the objects matched for height in the image. In the third experiment, we varied the orientation of the planes from a ground/ceiling orientation to a sidewall orientation, in small steps. To show that these results are not limited to artificial displays, we conducted a demonstration using direct vision of real objects. The fourth experiment considered the effects of height on the object at which distances are compared. The fifth experiment replicated the fourth experiment using planar ellipses instead of solid rectangular posts as the objects.

\section{EXPERIMENT 1 Ground/Ceiling and Sidewall Comparisons}

\section{Method}

Observers. The observers were 4 graduate students at the University of California, Irvine, ranging in age from 25 to 32, who were paid for their participation. All were naive regarding the purpose of the experiment, and all had normal or corrected-to-normal visual acuity (20/40 measured with a Snellen eye chart).

Stimuli. The stimuli were computer-generated 3-D scenes composed of a textured room and two posts. The texture scale on the ground and ceiling was different from that on the sidewalls to keep adjacent surfaces distinct. The simulated dimensions of the room were $240 \mathrm{~cm}$ wide $\times 240 \mathrm{~cm}$ high $\times 1,429 \mathrm{~cm}$ deep (see Figure 2). The simulated distances of the observer to the closer and more distant ends of the room were $571 \mathrm{~cm}$ and 2,000 cm, respectively. (The calculation of the scene dimensions was based on an eye-height of $120 \mathrm{~cm}$. )

Two textured posts were positioned $1,000 \mathrm{~cm}$ from the observer with dimensions of $32 \mathrm{~cm}$ wide $\times 192 \mathrm{~cm}$ high $\times 20 \mathrm{~cm}$ deep. The separation between the posts was $32 \mathrm{~cm}$. In half of the trials, the posts were centered horizontally in the display. One of the posts was in contact with the ground in the simulation, and there was a gap between its top surface and the ceiling. It was thus in optical contact with the ceiling, but not in physical contact with the ceiling. The other post was in contact with the ceiling but there was a gap between its bottom surface and the ground, so that it was in optical contact but not in physical contact with the ground (see Figures $2 \mathrm{~A}$ and $2 \mathrm{~B}$.) Thus, ground and ceiling contact provided contradictory information about the positions of the posts in the scene. In the other half of the trials, the posts were horizontal (rotated $90^{\circ}$ relative to the vertical posts), and both were centered vertically in the display. One post was in physical contact with the left wall in the simulation, but there was a gap between its right edge and the right wall. The other post was in physical contact with the right wall, but there was a gap between its left surface and the left wall (see Figures $2 \mathrm{C}$ and $2 \mathrm{D}$.) In these displays, optical contact with the right wall and left wall provided contradictory information about the positions of the posts in the simulation.

Design. There were two independent variables: (1) the pair of surfaces that were in contact with the posts, either the ground plane and the ceiling (ground/ceiling condition), or the right and left walls (right/left wall condition) and (2) the post that had the closer optical contact with the ground, either the right or left post in the ground/ceiling condition or the top or bottom post in the right/left wall condition. Which pair of surfaces had the larger texture elements was counterbalanced across trials. Each observer received six replications of each of the four combinations of the two independent variables, or 12 trials for each pair of surfaces.

In order to keep the type of response (right/left vs. top/bottom) consistent within blocks of trials, the ground/ceiling and right/left wall trials were presented in separate blocks. The first and the third block each contained 6 trials of one type, and the middle block contained 12 trials of the other type, with the order of the two trial types - ground/ceiling and right/left wall — counterbalanced across observers. The order of trials within a block was randomized. Four practice trials of the same type were added at the beginning of the first and the second blocks, and two practice trials were added at the beginning of the last block. Thus, the total number of trials in each block was 10,16 , and 8 , respectively. The observers were given a short break between blocks.

Apparatus. The displays were presented on a 21-in. (53-cm) flat screen CRT monitor with a pixel resolution of $1,280 \times 1,024$, controlled by a Windows 2000 workstation. The dimensions of the displays on the monitor were $30 \mathrm{~cm}$ wide $\times 30 \mathrm{~cm}$ high. A $19-\mathrm{cm}$ diameter collimating lens, which magnified the images by approximately $19 \%$, was located between the observer and the monitor. The distance between the observers' eyes and the lens was approximately $10 \mathrm{~cm}$, and the distance from their eyes to the monitor was $85 \mathrm{~cm}$. A chinrest was mounted at a position appropriate to this viewing distance.

Procedure. The experiment was run in a darkened room. The observers viewed the monitor binocularly through the collimating lens with their head in the chinrest. In each trial, a scene was displayed continuously for $30 \mathrm{sec}$. In the ground/ceiling condition, the observers were instructed to press either the right or left button of a mouse to indicate that either the right or left post appeared closer. In the right/left wall condition, the mouse was rotated $90^{\circ}$ counterclockwise. The observers pressed either the top or bottom button of the mouse to indicate that either the top or bottom post appeared closer. The observers were instructed to keep pressing a button until they found the other post to be closer, in which case they were to re- 

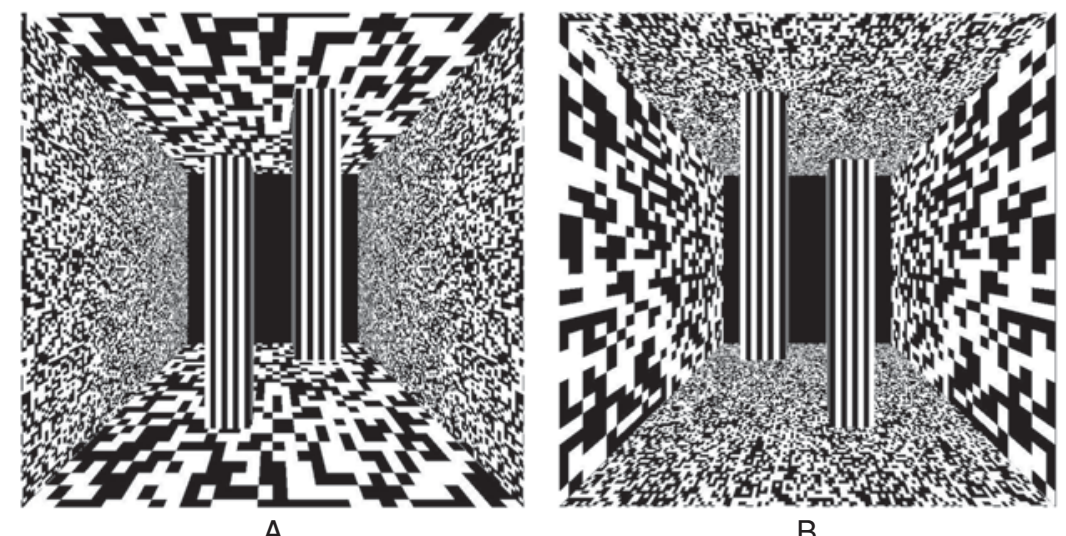

A

B

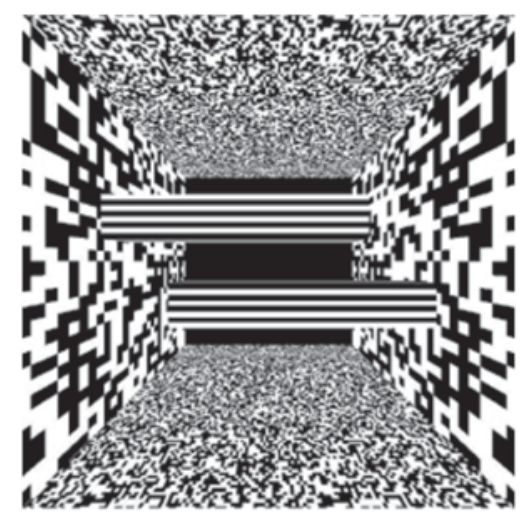

C

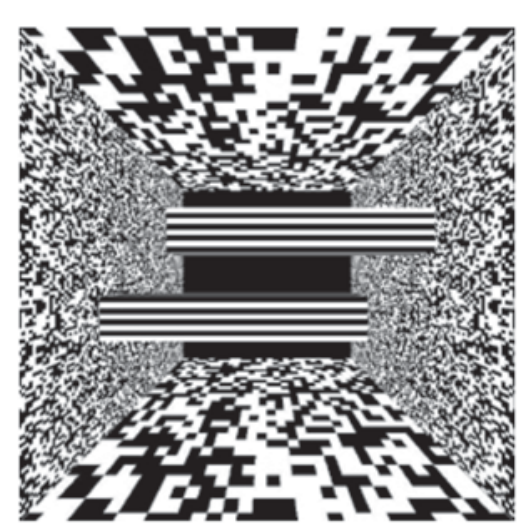

D

Figure 2. Examples of the stimuli in Experiment 1: (A) vertical posts with the left post attached to the ground and the right post attached to the ceiling, (B) vertical posts with the left post attached to the ceiling and the right post attached to the ground, (C) horizontal posts with the upper post attached to the left wall and the lower post attached to the right wall, and (D) horizontal posts with the lower post attached to the left wall and the upper post attached to the right wall.

lease the button they were pressing and then press the other button of the mouse. They were told to have a button pressed at all times during a trial and that they could change their choice as frequently as necessary. In each trial, the total time each button was pressed was recorded.

\section{Results and Discussion}

The observers' responses were classified as being in accord with one surface or another (ground or ceiling, right or left wall) if the post that the observer indicated was closer had the closer optical contact with that surface. The proportion of time that each observer's responses were in accord with each surface in each of the two surface-pair conditions is shown in Table 1. In the ground/ceiling condition, all 4 observers responded more often in accordance with the optical contact information for the ground surface than with that of the ceiling. For 2 observers, this proportion was 1.00 , and for the other 2 it was .74 and .84. In the right/left wall condition, responses in accordance with optical contact with the two walls showed little difference, with the proportion of re- sponses in accord with the optical contact information from each surface close to .50 for all 4 observers.

We compared the proportions in Table 1 in the ground/ ceiling conditions with those in the right/left wall conditions using a separate $t$ test for dependent samples for each observer. The proportions compared were the highest in each condition (the ground surface proportion for the ground/ceiling condition and either the right or left wall proportion for the right/left wall condition). All but

Table 1

Proportion of Time Spent Responding According to Each Surface

\begin{tabular}{cccccc}
\hline & \multicolumn{2}{c}{ Ground vs. Ceiling } & & \multicolumn{2}{c}{ Left Wall vs. Right Wall } \\
\cline { 2 - 3 } \cline { 5 - 6 } Observer & Ground & Ceiling & & Left Wall & Right Wall \\
\hline J.C. $^{*}$ & 1.00 & .00 & & .56 & .44 \\
X.L. & 1.00 & .00 & & .50 & .50 \\
P.Z. & .74 & .26 & & .47 & .53 \\
N.S. & .84 & .16 & & .48 & .52 \\
\hline
\end{tabular}

*Significant at .05 level. 
the third observer $(p=.1)$ showed a significant difference $(p<.05)$ between the proportion of choices corresponding to the ground plane and the highest proportion corresponding to the right or left wall. A $t$ test for dependent samples for all the observers pooled together found the difference between the highest proportions in the two conditions $(89.5 \%$ vs. $53.75 \%)$ to be significant $[t(3)=5.66, p<.05]$.

This result showed that when the ground plane and ceiling provide contradictory layout information in the scene, observers use the information from the ground more frequently than that from the ceiling. We will refer to this effect as a ground dominance effect. When the surfaces were sidewalls, observers did not show a preference for either surface.

Most observers showed a preference for judging the lower horizontal post as being closer, however, in the sidewall condition. The proportion of time when the lower post was judged as being closer was .77, 1.00, .76, and .43 for Observers J.C., X.L., P.Z., and N.S., respectively. The tendency to judge the lower horizontal post as being closer is consistent with a height in the visual field cue to depth (e.g., Sedgwick, 1986) and a bias for preferential processing of the lower visual field (e.g., Gysen, Verfaillie, \& De Graef, 2002).

\section{EXPERIMENT 2 \\ Equating Height in the Image}

In Experiment 1, the height of the posts in the image covaried with their optical contact positions on the ground surface. That is, the post indicated as being closer by the ground contact information was always lower in the image than the post indicated as being farther away. In Experiment2, we examined whether the ground dominance effect would occur based only on optical contact information, without the additional information provided by the difference in the height of the posts in the image. In this experiment, we produced displays in which the height of the two posts in the image was the same by tilting the ground plane and the ceiling. Sidewalls were not included.

\section{Method}

Observers. Two of the observers in Experiment 1, J.C. and X.L., participated.

Stimuli. The stimuli differed from those in Experiment 1 in two ways: (1) Only a ground plane and a ceiling plane were present in the displays, thus forming an open textured field, and (2) the field was tilted $15^{\circ}$ either to the right or left in each trial, making the two posts the same height in the image. Examples of the stimuli are shown in Figure 3.

Design. The independent variable was the post that had closer optical contact with the ground, either the right or left post. To keep the heights of the two posts in the image equal, the direction of tilt of the surfaces was determined by which post had the closer optical contact with the ground. The other post had closer optical contact with the ceiling. Half of the displays used large texture elements, and half used small texture elements. Each level of the independent variable was repeated six times, resulting in a total of 12 trials that were randomized for each observer. The experimental trials were preceded by 4 practice trials, one for each combination of the two levels of the independent variable with the two texture sizes. All trials were run in a single block.

Apparatus and Procedure. The apparatus and procedure were the same as in the first experiment. In each trial, the display was shown continuously for $30 \mathrm{sec}$. The observers were asked to keep pressing the button that corresponded to the post that appeared closer.

\section{Results and Discussion}

Both observers selected the post with a closer optical contact with the ground plane as being closer $100 \%$ of the time on every trial, which showed that the optical contact information could operate independently of the height of the posts in the image. Of course, tilting the ground surface and ceiling still left a difference in the height of the posts relative to the texture gradient of the ground plane. However, this difference is an essential element of optical contact with the ground plane, as suggested by both Gibson (1950) and Epstein (1966).
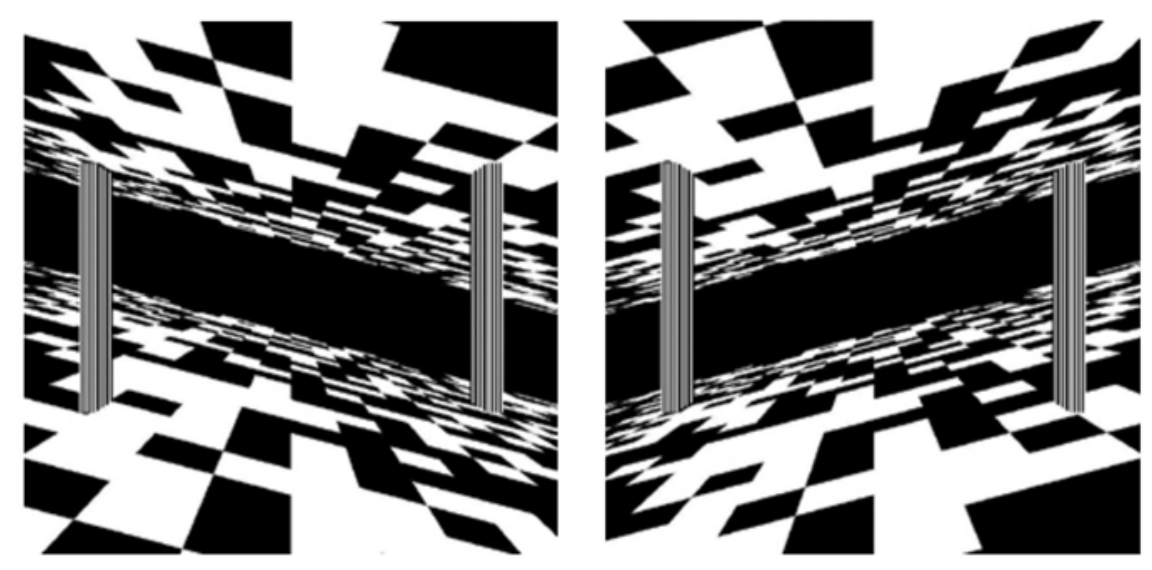

Figure 3. Examples of stimuli used in Experiment 2. 


\section{EXPERIMENT 3 Intermediate Surface Tilts}

In the first two experiments, we found that when optical contact with the ground and the ceiling provided contradictory layout information, observers responded according to the information provided by the ground surface. When the surfaces were two sidewalls, however, observers did not show a bias. The sidewall displays were $90^{\circ}$ rotations of the ground/ceiling displays, and this raises the question of whether the ground dominance effect gradually diminishes for rotations of the scene between $0^{\circ}$ and $90^{\circ}$, or whether there is an intermediate rotation angle at which ground dominance is no longer observed. In Experiment 3, we addressed this question by presenting scenes at intermediate levels of rotation, in addition to ground/ceiling and right/left wall scenes.

\section{Method}

Observers. The observers were 7 graduate students at the University of California, Irvine, ranging in age from 25 to 32, who were paid for their participation. All met the same visual-acuity requirements as the observers in Experiment 1. All were naive regarding the purpose of the experiment, and none had participated in Experiment 1 or 2 .

Stimuli. The stimuli were scenes consisting of a ground plane, a ceiling, and two posts. The scene was shown with the ground/ceiling orientation used in Experiment 1, which we labeled as $0^{\circ}$ tilt, or tilted either clockwise or counterclockwise. The tilt ranged from $-75^{\circ}$ to $90^{\circ}$ in steps of $15^{\circ}$, with negative values representing counterclockwise rotations. Examples of the stimuli are shown in Figure 4 . We did not include a $-90^{\circ}$ condition because its image was the same as that of the $90^{\circ}$ condition.
Design. The independent variable was the tilt of the surfaces $\left(-75^{\circ},-60^{\circ},-45^{\circ},-30^{\circ},-15^{\circ}, 0^{\circ}, 15^{\circ}, 30^{\circ}, 45^{\circ}, 60^{\circ}, 75^{\circ}\right.$, or $\left.90^{\circ}\right)$. There were 20 trials at each level of tilt. Which of the two posts had the closer ground contact was counterbalanced across trials. The 240 trials were divided into two blocks with 10 repetitions of each tilt level in each block. These experimental trial blocks were preceded by a 48 -trial practice block with 4 repetitions of each tilt level. The order of the trials for each observer in each block was randomized individually.

Apparatus and Procedure. The apparatus and procedure were the same as in Experiment 1, with the following exceptions: On each trial, the observer viewed the display monocularly through a $30-\mathrm{cm}$ diameter circular aperture behind the collimating lens. The circular aperture was intended to keep the distances between the borders of the visible image and such features as the tops and bottoms of the posts similar across different tilts. A black background was first displayed on the screen for $1,000 \mathrm{msec}$, after which a white cross mark appeared in the center of the screen. After $500 \mathrm{msec}$, the display with the two planes and two posts was shown with the mouse cursor (an arrow) in the center of the display. The task of the observer was to judge which post appeared closer and to respond by moving the cursor onto that post and clicking the left button of the mouse. The observers were encouraged to respond as soon as they had made a choice. If the cursor was not positioned near one of the two posts when the mouse was clicked, or if the observer did not respond within $5 \mathrm{sec}$, that trial was repeated at the end of the block. The observer's selection and response time were recorded on each trial. The reason for changing the response method and display duration from Experiments 1 and 2 was that we were mainly interested in the observer's initial perception of the scene in this experiment, rather than how that perception changed over time.

\section{Results and Discussion}

We calculated the proportion of trials on which the post with closer optical contact with the ground was se-

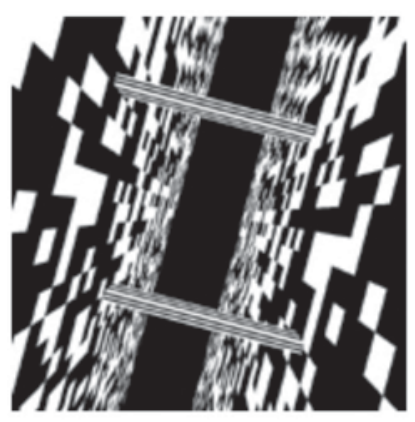

$-75^{\circ}$

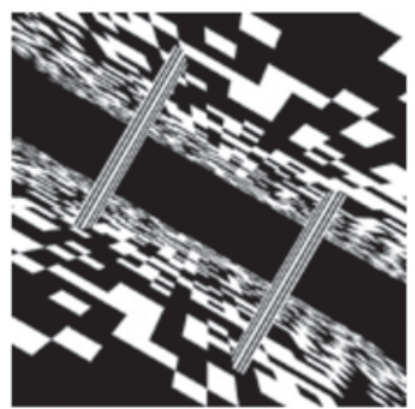

$30^{\circ}$

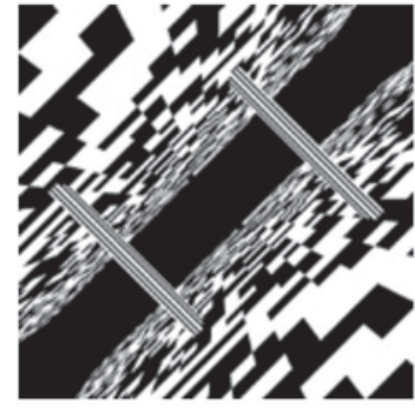

$-45^{\circ}$

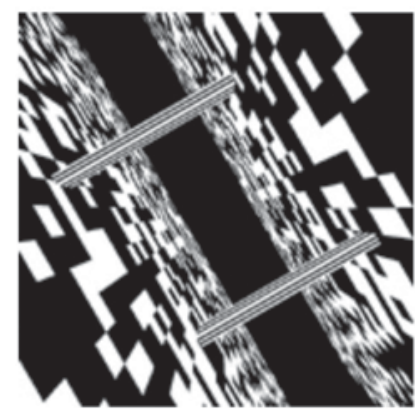

$60^{\circ}$

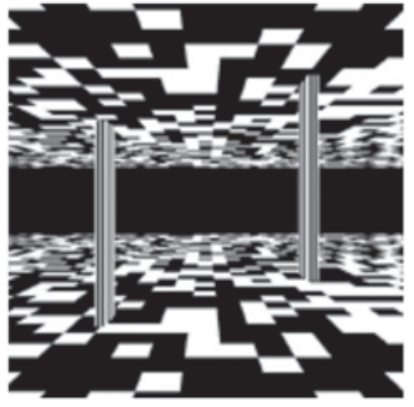

$\mathbf{0}^{\mathbf{0}}$

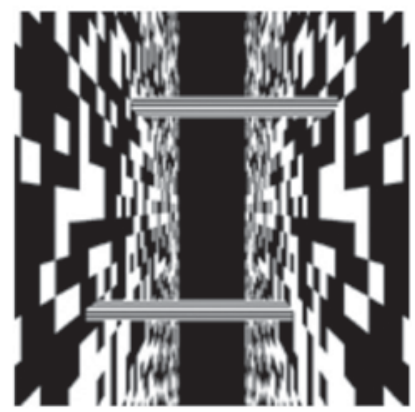

$90^{\circ}$

Figure 4. Examples of stimuli used in Experiment 3. 
lected. Because there was no basis for labeling one of the planes as a ground plane in the $90^{\circ}$ condition, the left wall was arbitrarily labeled as the "ground" in that condition.

As the tilt of the surfaces increased from $0^{\circ}$ to $90^{\circ}$ in either direction, the proportion of trials on which responses were in accordance with the ground surface dropped gradually, from almost $100 \%$ to around $75 \%$, as can be seen in Figure 5. This shows that the reliance on optical contact information from a surface in layout judgments gradually diminishes as the orientation of this surface deviates from the typical orientation of a ground surface. A two-way analysis of variance (ANOVA) showed a significant main effect of the tilt angle $[F(11,66)=$ $3.94, p<.01]$. As expected, the main effect of which of the two posts had a closer optical contact with the ground surface did not reach significance $[F(1,6)<1, p>.05]$, and the interaction was not significant. In the $90^{\circ}$ condition, although we had expected the observers to use the information from the left and right walls equally, we found that they selected the post that had a closer optical contact with the left wall more frequently (around 73\%). Since the ground surface and the ceiling were symmetrical and there was no way the observers could recognize which wall was labeled as ground and which was the ceiling, this can only be explained by a preference for responding in accordance with optical contact with the left wall in this condition. This preference was found for 3 of the 7 observers, who responded according to contact with the left wall on $90 \%$ to $100 \%$ of the trials. The remaining 4 observers responded according to contact with the left wall on $40 \%$ to $75 \%$ of the trials. (If we had scored half of the $90^{\circ}$ trials with the left wall as ground and half with the right wall as ground, the proportion for that condition would have been .5.)
We also conducted a two-way ANOVA for the response time $(\mathrm{RT})$ and found a significant main effect $[F(11,66)=$ $6.04, p<.001]$ for the tilt angle. The main effect of which of the two posts had the closer ground contact did not reach significance $[F(1,6)=1.10, p>.05]$, and the interaction was not significant. In Figure 6 , we can see that the RTs for conditions around $0^{\circ}$ were shortest and increased with increasing tilt of the surfaces.

These three experiments together establish the dominance of the ground surface in determining layout in computer-simulated scenes. Direct vision, however, may provide depth cues that are not available in simulated displays or pictures, whereas computer displays may contain flatness cues that are not present in direct vision. For this reason, it is important to determine whether we can find the same effect with direct viewing of a real 3-D scene.

\section{“REAL-WORLD” DEMONSTRATION}

\section{Method}

Observers. The observers were 26 undergraduate students at the University of California, Irvine, who received course credit for their participation. All had visual acuity of at least $20 / 40$ or corrected to 20/40.

Stimuli. The experiment was conducted in a corridor of the Social Science Laboratory Building at the University of California, Irvine (Figure 7). The dimensions of the corridor were $1.85 \mathrm{~m}$ wide $\times 2.59 \mathrm{~m}$ high $\times 15.24 \mathrm{~m}$ deep. Two wooden posts, both painted red, were positioned in the corridor. The dimensions of each post were $0.08 \mathrm{~m}$ wide $\times 0.04 \mathrm{~m}$ deep $\times 2.36 \mathrm{~m}$ high. One was attached to the ground and was $0.23 \mathrm{~m}$ below the ceiling; the other was attached to the ceiling and was $0.23 \mathrm{~m}$ above the ground. Each post was $0.28 \mathrm{~m}$ from the closer sidewall. The separation between the posts was $1.14 \mathrm{~m}$. The corridor was well lighted, except that the light on the ceiling immediately behind the posts was turned off in order to reduce the visibility of shadows. For the same reason, we

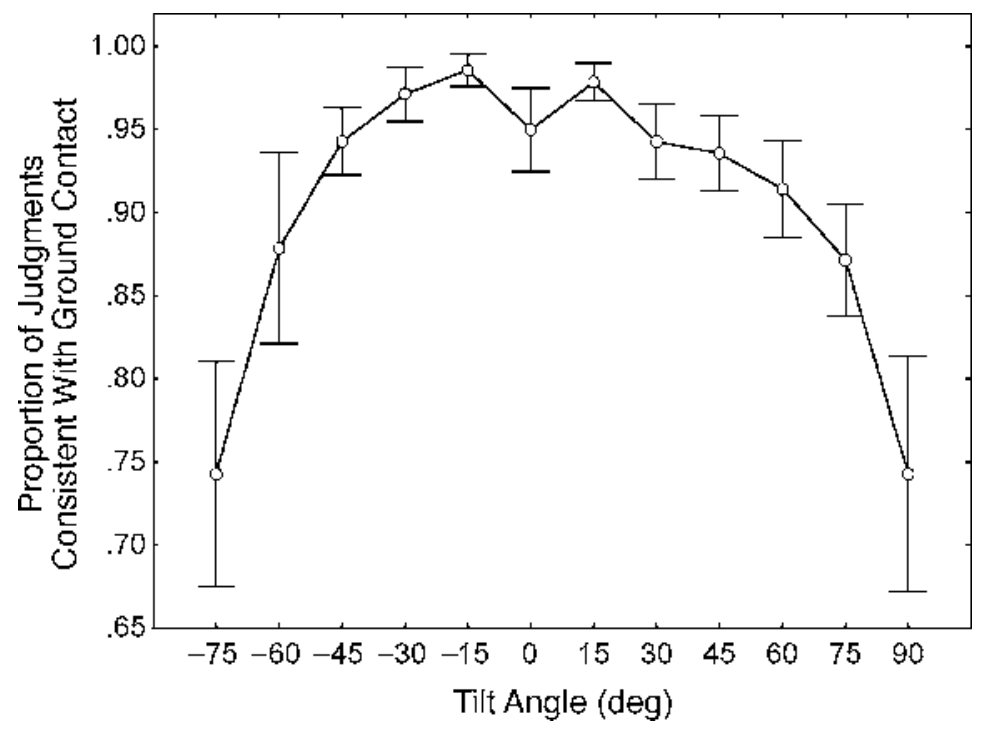

Figure 5. The effect of the tilt angle on the proportion of trials on which the post with closer optical contact with the ground was selected (Experiment 3). Error bars represent \pm 1 standard error after centering. (Centering removes effects of differences in the overall means across subjects.) 


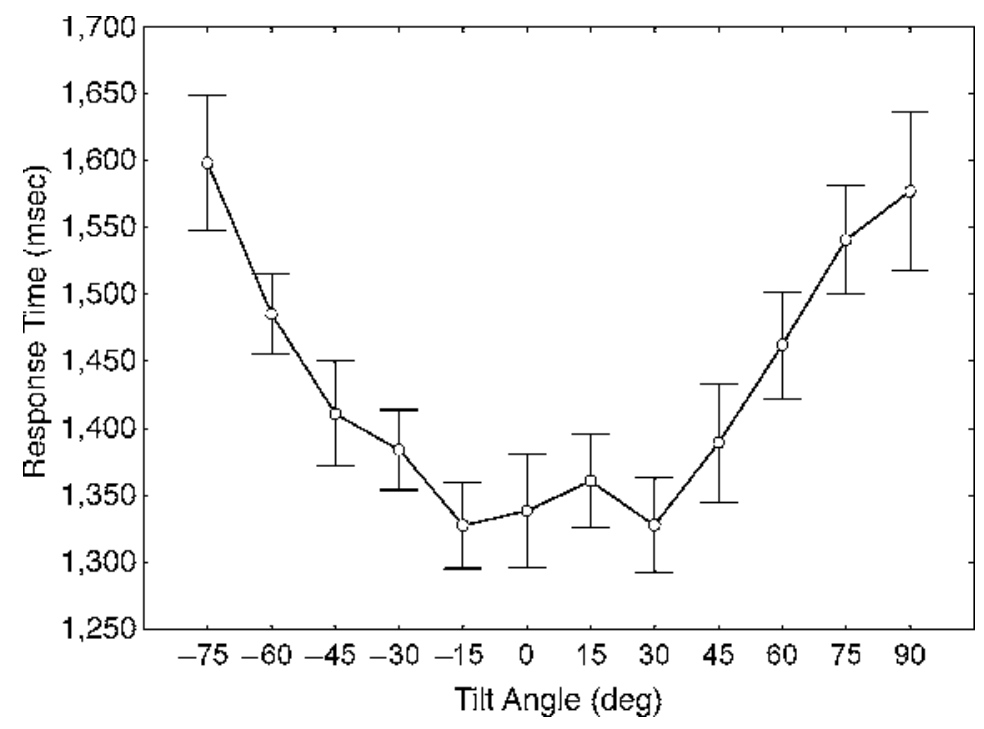

Figure 6. The effect of the tilt angle on response time (Experiment 3). Error bars represent \pm 1 standard error after centering.

put small lights in the cable trays that were just below the top of the posts on both sidewalls. A table with a chinrest was located $9.75 \mathrm{~m}$ from the posts.

Design. One independent variable was manipulated in this experiment: which post had the closer optical contact with the ground surface, either the right post or the left post. We used a betweensubjects design in which each observer viewed either one of the two conditions once and made a single judgment of which of the two posts appeared to be closer to him or her.

Procedure. The displays were viewed monocularly from a distance of $9.75 \mathrm{~m}$. The observer sat in a chair, put an eye patch over his or her nonpreferred eye and rested his or her chin on the chinrest. Monocular viewing with the head stationary was used because binocular disparity and motion parallax are likely to affect perceived distances at this viewing distance. The display was at first hidden from the observers by a dark curtain. After the experimenter opened the curtain, the observer had $5 \mathrm{sec}$ to see the display and was instructed to verbally report as soon as possible which post appeared to be closer. If the observer did not respond within $5 \mathrm{sec}$, the curtain was closed but the observer was still allowed to respond.

\section{Results and Discussion}

Overall, 22 of 26 observers selected the post that had the closer optical contact with the ground (floor) as being closer. Specifically, when the right post was attached to
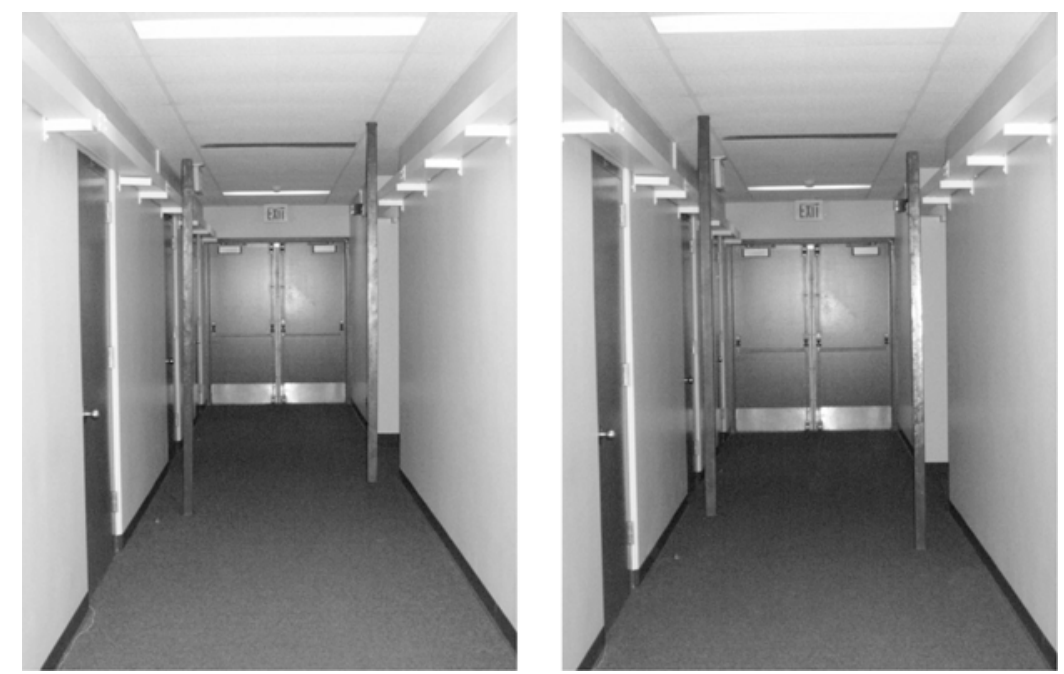

Figure 7. The stimuli used in the real-world demonstration. In the left picture, the left post was attached to the ground; in the right picture, the right post was attached to the ground. 
the ground, 12 of 13 observers chose it as being closer. When the left post was attached to the ground, 10 of 13 observers chose it as being closer. The proportion of observers choosing the post with optical contact with the ground was significantly different from chance $[.5 ; p<$ .01 (two-tailed binomial test)]. Although the ground and ceiling conditions could not be matched exactly in this demonstration (for example, the ground was carpeted, whereas the ceiling had acoustic tiles), the results suggest that the dominance of the ground surface in determining perceived layout exists with direct vision of real scenes.

\section{EXPERIMENT 4 Location of the Judgment}

Our interpretation of the results of the first three experiments is that layout is perceived primarily in accordance with ground contact. There is another possible interpretation, however. The bottom of the post may be perceived in accordance with ground contact, and the top of the post may be perceived in accordance with ceiling contact, leading to a perception of the post as being slanted in depth. When asked to judge the relative distance of two posts, observers may have compared the distances at the bottom of the posts, resulting in judgments corresponding to ground contact. In Experiment 4, we examined the possibility that the ground dominance effect observed in the first three experiments was due to a preference for judging relative distances at the bottoms of objects in a scene. Observers were asked to judge the relative distance of two markers, one on each post, rather than the relative distance of the posts. The markers were positioned at various heights on the posts. If the posts were perceived as being slanted in depth, we would expect the proportion of responses made in accordance with ground contact to drop from $100 \%$ when the judgments were made at the bottom of the posts to around $0 \%$ when the judgments were made at the top of the posts. ${ }^{1}$

\section{Method}

Observers. The observers were 5 graduate students at the University of California, Irvine, ranging in age from 25 to 32, who were paid for their participation. All had visual acuity of at least 20/40 or corrected to that level. All were naive to the purpose of the experiment. Two had participated in Experiment 3.

Stimuli. The scene was similar to that used in Experiment 2, with a ground plane and a ceiling tilted either to the right or left and two vertical posts at the same height in the image. A blue dot, $5.6 \mathrm{~cm}$ in diameter, was attached to each of the two posts at the same height, as shown in Figure 8. The location of the dots varied from near the bottom to near the top of the posts, in five equal steps.

Design. There were two independent variables: (1) the post that was indicated as closer by the ground surface (the right or left post); and (2) the location of the dots on the posts (bottom, mid-bottom, middle, mid-top, and top). This produced 10 combinations, each of which was repeated four times in the first block of trials and six times in the second block. Twenty practice trials with two repetitions of each combination preceded the first block. The order of the trials was randomized for each observer in each block.

Apparatus and Procedure. The apparatus and procedure were the same as in Experiment 3, with the following exceptions. On each trial, the observer viewed the display monocularly through a collimating lens. A black background was first displayed for
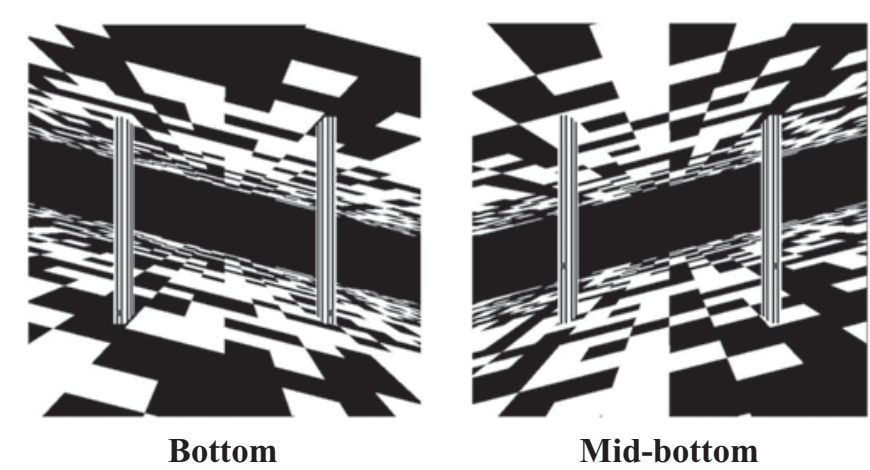

Mid-bottom

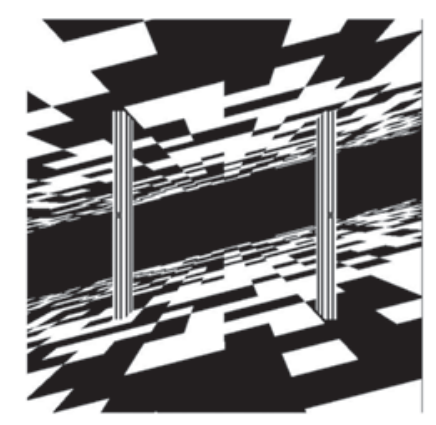

Middle
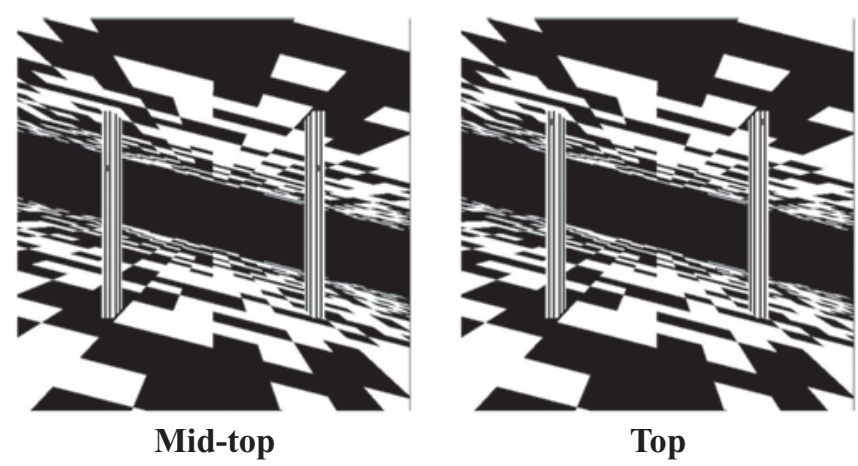

Figure 8. Examples of stimuli used in Experiment 4. 
$1,000 \mathrm{msec}$, after which a white cross appeared in the center of the black field. After $500 \mathrm{msec}$, the display with the two planes, two posts and two dots appeared, with a cursor positioned in the center. The observer's task was to judge which of the two dots appeared closer by moving the cursor onto that dot and clicking the left button of the mouse. Note that this is different from the previous task in which the observer was asked to judge which post was closer. The observers were encouraged to respond as soon as they had made a choice. If the cursor was not positioned close to one of the dots when the mouse was clicked, or if the observer did not respond within $6 \mathrm{sec}$, that trial was repeated at the end of the block. (The longer viewing time, $6 \mathrm{sec}$ vs. $5 \mathrm{sec}$ in Experiment 3, was introduced due to the increased difficulty of the task-clicking on a dot rather than on a post.) The observer's selection and RT were recorded on each trial.

\section{Results and Discussion}

The proportion of judgments based on ground contact information for each of the five dot positions is shown in Figure 9. A two-way ANOVA revealed a significant main effect of the location of the dots $[F(4,16)=7.58, p<$ $.01]$. The main effect of which of the two posts had a closer optical contact with the ground did not reach significance $[F(1,4)=0.34, p=.59]$, and the interaction was not significant. When the dots were at the bottom of the posts, this proportion was close to 1.0 , which means that almost all of the judgments were consistent with optical contact for the ground surface, rather than the ceiling surface. As the dots moved from bottom to top, this proportion dropped continuously to around $74 \%$, which still shows a preference to use the ground surface, rather than the ceiling surface, to judge the layout of the scene. This result would not be expected if the distance of the bottom of the post was perceived according to ground contact and the top of the post was perceived according to ceiling contact. Although there is some reduction in the percentage of choice made in accordance with ground contact as the dots are moved higher on the post, it does not drop to $0 \%$ even when the dots are at the top of the post.

Figure 10 shows RT as a function of the location of the dots on the post. A two-way ANOVA revealed a significant main effect of dot location $[F(4,16)=5.70, p<$ .01]. The main effect of which of the two posts had the closer ground contact was not significant $[F(1,4)=0.93$, $p=.39]$. The interaction was not significant. As we can see from Figure 10, the RT when the dots were higher on the posts was generally greater than when they were lower. This increase in RT with increasing height is consistent with McCarley and He's (2000) finding that the RT in a visual search task was faster when the target was located on a ground surface than when it was on a ceiling surface.

\section{EXPERIMENT 5 Ellipses}

The results of Experiment 4 indicate that the ground dominance effect was not due to the posts being perceived as slanted, but it is possible that they would have been perceived that way if not for the rectangular shape of the posts and the regular stripe texture. Those two related factors would have been expected to provide a strong indication that the posts were upright. In order to minimize the effect of the shape of the posts and remove any effect of the regular texture, we replicated Experiment 4 with the posts replaced by gray ellipses. Ellipses provide a much weaker cue to orientation than do rectangles (e.g., Braunstein \& Payne, 1968).

\section{Method}

Observers. The observers were 4 graduate students at the University of California, Irvine, who were paid for their participation. All had a visual acuity of at least $20 / 40$ or corrected to that level.

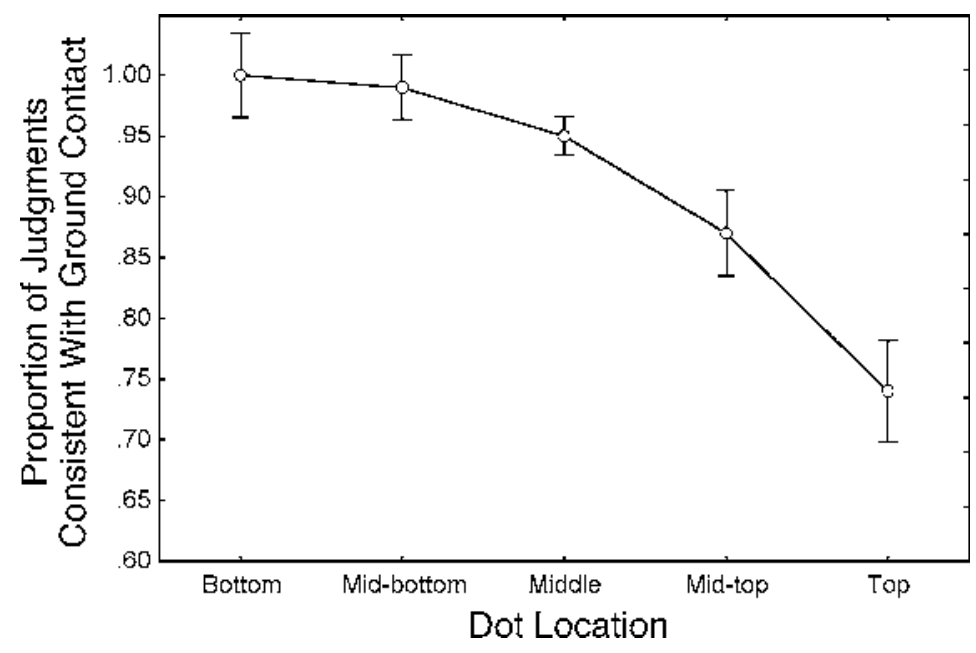

Figure 9. The effect of the location of the dots on the proportion of judgments based on ground contact information (Experiment 4). Error bars represent \pm 1 standard error after centering. 


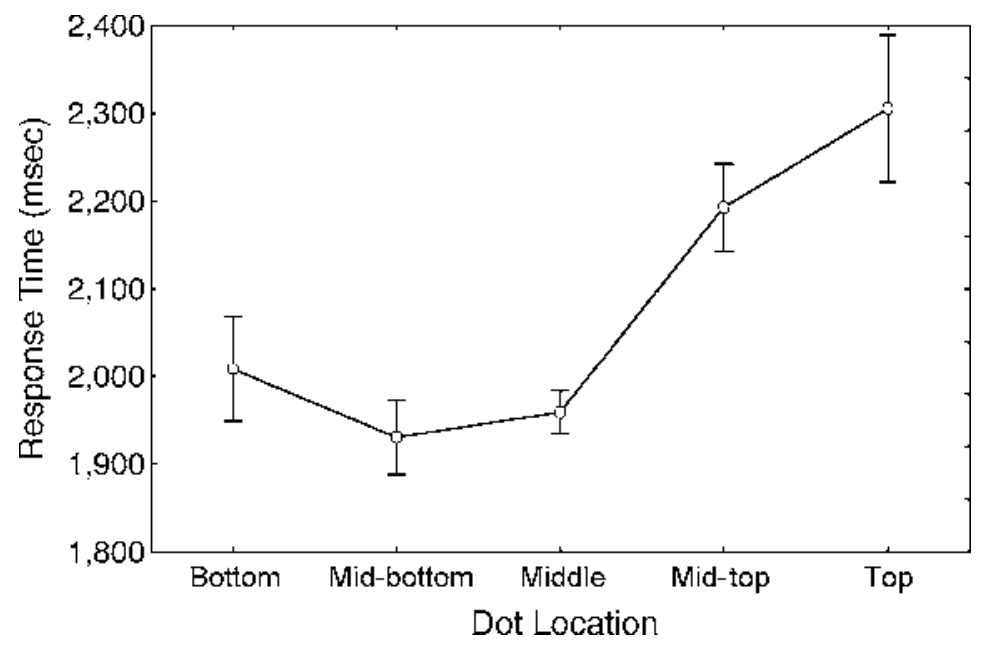

Figure 10. The effect of the location of the dots on response time (Experiment 4). Error bars represent \pm 1 standard error after centering.

All had participated in Experiment 4 but were naive to the purpose of the experiment.

Stimuli, Design, Apparatus, and Procedure. The stimuli were the same as those in Experiment 4, except that the posts were replaced with gray ellipses, as shown in Figure 11. The design, apparatus, and procedure were the same as in Experiment 4.

\section{Results and Discussion}

The results were similar to those in Experiment 4. In Figure 12, we can see that the proportion of judgments agreeing with optical contact with the ground surface was about .93 and dropped gradually as the dots were located higher on the ellipses. But even when the dots were at the top of the ellipses, this proportion was still .82, showing a dominance of the ground surface in determining layout judgments. This was consistent with Experiment 4, although a two-way ANOVA did not indicate a significant main effect of the location of the dots $[F(4,12)=1.92, p=.17]$. The main effect of which of the two posts had a closer optical contact with the ground was not significant $[F(1,3)=1.96, p=.26]$. The interaction was not significant.

Figure 13 shows an increase in RT as the dots moved from the bottom to the top of the ellipses, which was also consistent with the results of Experiment 4. A two-way ANOVA revealed a significant main effect of the location of the dots $[F(4,12)=7.99, p<.01]$. The main effect of which of the two posts was indicated by the ground as being closer was not significant $[F(1,4)=1.32, p=$ .33]. The interaction was not significant. Overall, the results of Experiments 4 and 5 indicate that the ground dominance effect cannot be explained by the location of the judgment.

\section{GENERAL DISCUSSION}

In this series of experiments, we examined the dominance of the ground surface over other environmental surfaces, especially the ceiling surface, in indicating perceived layout in 3-D scenes. We found when the ground surface and the ceiling surface provided contradictory optical contact information for two vertical posts that were at the same simulated distance, observers preferred to use the ground surface as the basis for judging layout, but when the two surfaces were sidewalls, most observers did not show a preference for either surface. This result is consistent with the hypothesis that the ground surface, compared with other environmental surfaces, has a special importance in organizing 3-D scenes.

When the tilt of the surfaces was varied in $15^{\circ}$ steps from a ground/ceiling orientation to a sidewall orientation, there was a gradual decrease in the ground dominance effect. Ground dominance persisted, however, even when the scene was tilted more than $45^{\circ}$, and the surfaces were thus closer to a sidewall orientation than to a ground/ceiling orientation. This suggests a preference to treat one of the surfaces as the ground plane whenever possible. RT increased as the scene was tilted away from a ground/ceiling orientation. This is consistent with a mental rotation of the planes to a ground/ceiling orientation (e.g., Shepard \& Metzler, 1971), but it could also reflect an increase in the difficulty of judgment when the planes were tilted.

We also examined whether the ground dominance effect was due to a preference to judge the relative distances of the posts at a specific location (e.g., the lower part) of the posts. Although the proportion of judgments corresponding to ground contact was reduced as the judgments were made higher on the post, most judgments were made in accordance with ground contact even when the positions being judged were at the top of the posts. This result is not consistent with an explanation of ground dominance for these stimuli in which the top and bottom of the posts were perceived as having different relative distances, but observers used the distances 


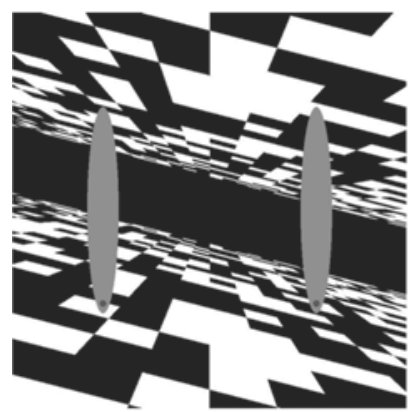

Bottom

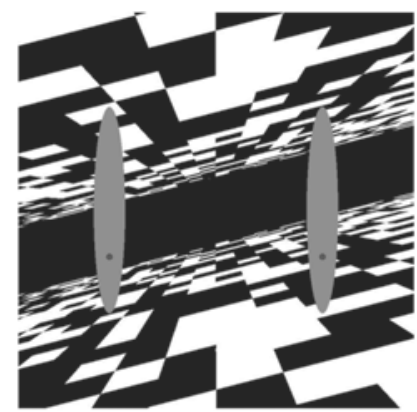

Mid-bottom

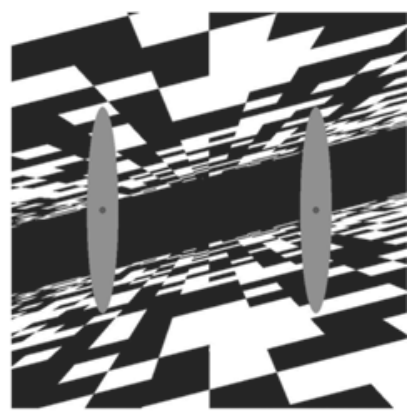

Middle

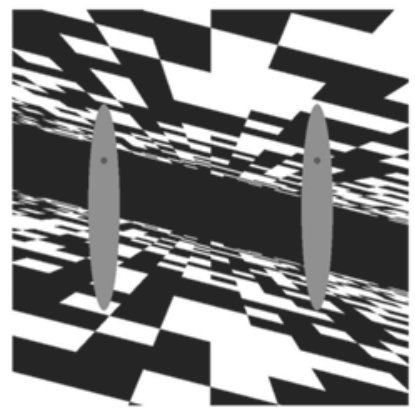

Mid-top

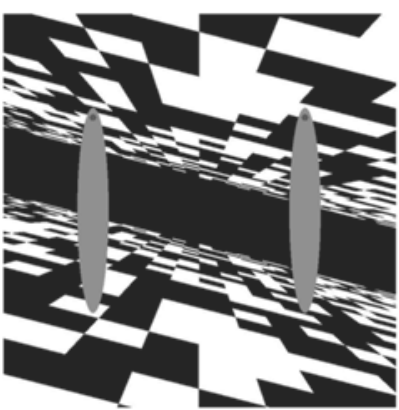

Top

Figure 11. Examples of stimuli used in Experiment 5.

at the bottom when asked to judge which post was closer. That explanation would predict that when asked to judge distances at the top of the posts, the proportion of judgments made in accordance with ground contact would drop to nearly 0 , rather than the .75 observed. The results do indicate, however, that the location of the judgment is relevant. A possible explanation is that the influence of optical contact with the ceiling is increased as attention is drawn to the top of the post. (A change in fixation may also have been involved; we did not control eye movements in these experiments.)

Whereas previous studies (Feria et al., 2003; He \& Nakayama, 1992, 1994a, 1994b; Meng \& Sedgwick, 2001, 2002; Sinai et al., 1998) revealed the importance

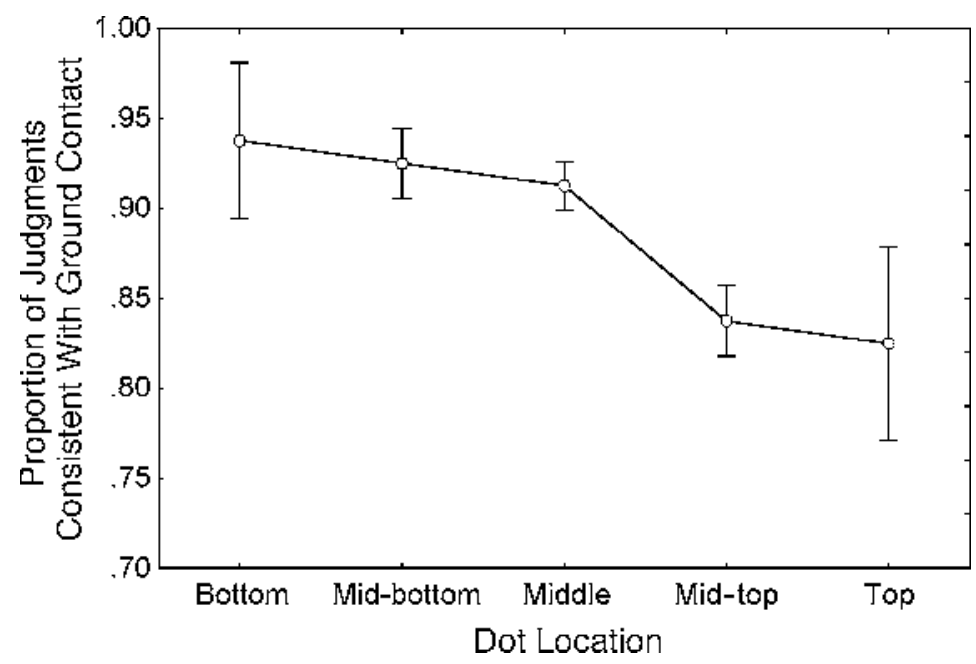

Figure 12. The effect of the location of the dots on the proportion of judgments based on ground contact information (Experiment 5). Error bars represent \pm 1 standard error after centering. 


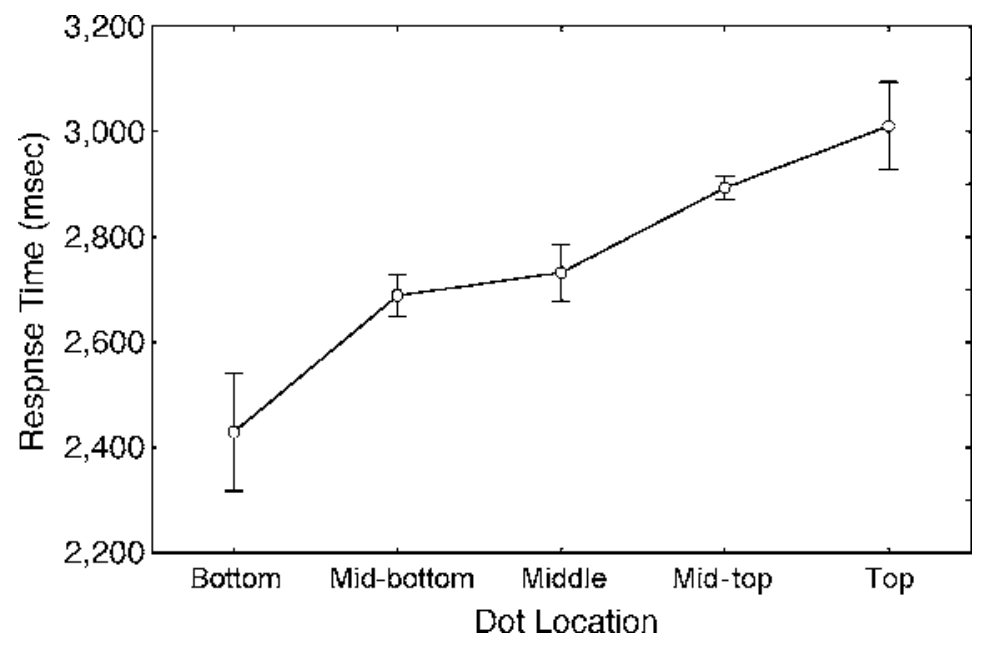

Figure 13. The effect of the location of the dots on response time (Experiment 5). Error bars represent \pm 1 standard error after centering.

of a surface, especially a ground surface, in organizing 3 -D scenes, our results, together with those of McCarley and $\mathrm{He}$ (2000), point to a unique role for the ground surface, among all environmental surfaces, in the perception of the visual world. However, the question of what leads to this dominance of the ground surface needs to be answered. An essential difference between a ground surface and a ceiling surface is that a ground surface recedes in depth from the bottom of its projected image to eye height, whereas a ceiling surface recedes in depth from the top of its projected image to eye height. Our visual system may have adapted to the layout of the ground surface, as suggested by Gibson (1950), Nakayama et al. (1995), and McCarley and He, thus processing information on a ground plane more efficiently than information on a ceiling surface.

However, there is another distinction between a ground plane and a ceiling: A ground plane is generally in the lower part of the visual field, whereas the ceiling is generally in the upper part of the visual field, for an upright observer. The ground dominance effect may reflect a bias to attend to the lower part of the visual field. Our results in Experiments 4 and 5, as mentioned above, are consistent with this hypothesis. This possibility is also consistent with the results of a recent figure-ground study by Vecera, Vogel, and Woodman (2002), in which observers were shown displays composed of two abutting irregular regions, either in an upper-lower orientation or in a left-right orientation, and asked to report which of the two regions appeared to be the figure. They found that in the right-left configuration, the two regions were equally likely to be chosen as the figure. However, in the upper-lower configuration, the lower region was more likely to be recognized as the figure than was the upper region, suggesting that the lower region is more salient than the upper region when presented at the same time. It should be noted that these two possible reasons for the ground dominance effect - whether recession in depth occurs from top to bottom or bottom to top and location in the visual field - are not necessarily mutually exclusive. It is possible that they both play a part in leading to the uniqueness of the ground surface, and their effects may interact with each other. This is a topic for future research.

Another question left unanswered in the present study is how the ground dominance effect is related to the frame of reference by which the ground is defined. In the research reported here, "up" and "down" would be the same whether a retinal, environmental, or gravitational reference frame was used. But these three frames of reference can be separated. Vecera (2004) compared a retinal reference frame with an environmental-gravitational reference frame in a study of top-bottom influences in figure-ground assignment. To separate the retinal reference frame from the environmental-gravitational reference frame, observers were asked to make judgments with their heads tilted sideways or upside down. The results were consistent with a viewer-centered reference frame, rather than an environmental or gravitational reference frame. Those results suggest that the ground dominance effect may also depend on a viewer-centered reference frame, but the figure-ground task and the layout task are quite different, and it is important to determine experimentally whether the ground dominance effect also depends primarily on retinal location, regardless of head orientation.

Overall, in this study we demonstrated the dominance of the ground surface over other environmental surfaces, especially the ceiling surface, in determining perceived layout both in a computer-simulated display and in a real scene. This effect decreases as the scene is tilted and does not depend on the height of the posts in the image. A preference to judge at a specific location of the posts could not account for this effect, since it existed, al- 
though in a lesser degree, even when the judgment was made at the top of the posts. These results demonstrate the uniqueness of the ground surface in organizing our perception of the visual world.

\section{REFERENCES}

Alhazen, I. (1989). Book of optics. In A. I. Sabra (Trans.), The optics of Ibn-Haytham (Vol. 1). London: University of London, Warburg Institute.

Andersen, G. J., Braunstein, M. L., \& Saidpour, A. S. (1998). The perception of depth and slant from texture in three-dimensional scenes. Perception, 27, 1087-1106.

Braunstein, M. L. (1968). Motion and texture as sources of slant information. Journal of Experimental Psychology, 78, 247-253.

Braunstein, M. L., \& Andersen, G. J. (1981). Velocity gradients and relative depth perception. Perception \& Psychophysics, 29, 145-155.

Braunstein, M. L., \& Payne, J. W. (1968). Perspective and the rotating trapezoid. Journal of the Optical Society of America, 58, 399-403.

Epstein, W. (1966). Perceived depth as a function of relative height under three background conditions. Journal of Experimental Psychology, 72, 335-338.

Feria, C. S., Braunstein, M. L., \& Andersen, G. J. (2003). Judging distance across texture discontinuities. Perception, 32, 1423-1440.

Gibson, J. J. (1946). Perception of distance and space in the open air. In J. J. Gibson (Ed.), Motion picture testing and research (Army Air Force Aviation Psychology Program Res. Rep. No. 7). [Reprinted in D. C. Beardslee \& M. Wertheimer (Eds.) (1958), Readings in perception. Princeton, NJ: Van Nostrand.]

GiBson, J. J. (1950). The perception of the visual world. Boston: Houghton-Mifflin.

Gibson, J. J. (1979). The ecological approach to visual perception. Boston: Houghton-Mifflin.

Gillam, B., FlagG, T., \& Finlay, D. (1984). Evidence for disparity change as the primary stimulus for stereoscopic processing. Perception \& Psychophysics, 36, 559-564.

Gysen, V., Verfaillie, K., \& De Graef, P. (2002). Transsaccadic perception of translating objects: Effects of landmark objects and visual field position. Vision Research, 42, 1785-1796.

He, Z. J., \& NaKayama, K. (1992). Surfaces versus features in visual search. Nature, 359, 231-233.

He, Z. J., \& NAKAYAMA, K. (1994a). Apparent motion determined by surface layout not by disparity or three-dimensional distance. Nature, 367, 173-175.

He, Z. J., \& Nakayama, K. (1994b). Perceiving textures: Beyond filtering. Vision Research, 34, 151-162.

He, Z. J., \& NaKaYAma, K. (1995). Visual attention to surfaces in 3-D space. Proceedings of the National Academy of Sciences, 92, 1115511159.

McCarley, J. S., \& HE, Z. J. (2000). Asymmetry in 3-D perceptual organization: Ground-like surface superior to ceiling-like surface. Perception \& Psychophysics, 62, 540-549.
Meng, J. C., \& Sedgwick, H. A. (2001). Distance perception mediated through nested contact relations among surfaces. Perception \& Psychophysics, 63, 1-15.

Meng, J. C., \& Sedgwick, H. A. (2002). Distance perception across spatial discontinuities. Perception \& Psychophysics, 64, 1-14.

Nakayama, K., He, Z. J., \& Shimojo, S. (1995). Visual surface representation: A critical link between lower-level and higher-level vision. In S. M. Kosslyn \& D. N. Osherson (Eds.), Visual cognition: An invitation to cognitive science (2nd ed., Vol. 2, pp. 1-71). Cambridge, MA: MIT Press.

NoRman, J. F., \& TodD, J. T. (1998). Stereoscopic discrimination of interval and ordinal depth relations on smooth surfaces and in empty space. Perception, 27, 257-272.

Rogers, B., \& Graham, M. (1979). Motion parallax as an independent cue for depth perception. Perception, 8, 125-134.

SEDGWICK, H. A. (1983). Environment-centered representation of spatial layout: Available visual information from texture and perspective. In J. Beck, B. Hope, \& A. Rosenfeld (Eds.), Human and machine vision (pp. 425-458). New York: Academic Press.

SEDGwick, H. A. (1986). Space perception. In K. R. Boff, L. Kaufman, \& J. P. Thomas (Eds.), Handbook of perception and human performance (pp. 21-1 to 21-57). New York: Wiley.

ShePARD, R. N., \& Metzler J. (1971). Mental rotation of threedimensional objects. Science, 171, 701-703.

SinAI, M. J., OoI, T. L., \& HE, Z. J. (1998). Terrain influences the accurate judgement of distance. Nature, 395, 497-500.

STEvens, K. A. (1981). The visual interpretation of surface contours. Artificial Intelligence, 217, 47-74.

Tittle, J. S., Norman, J. F., Perotti, V. J., \& Phillips, F. (1998). The perception of scale-dependent and scale-independent surface structure from binocular disparity, texture, and shading. Perception, 27, 147-166.

Todd, J. T., \& AKerstrom, R. A. (1987). Perception of three-dimensional form from patterns of optical texture. Journal of Experimental Psychology: Human Perception \& Performance, 13, 242-255.

Turner, J., Braunstein, M. L., \& Andersen, G. J. (1997). Relationship between binocular disparity and motion parallax in surface detection. Perception \& Psychophysics, 59, 370-380.

Vecera, S. P. (2004). The reference frame of figure-ground assignment. Psychonomic Bulletin \& Review, 11, 909-915.

Vecera, S. P., Vogel, E. K., \& Woodman, G. F. (2002). Lower region: A new cue for figure-ground assignment. Journal of Experimental Psychology: General, 131, 194-205.

\section{NOTE}

1. We thank Stephen Palmer for calling this issue to our attention at the 2003 Psychonomic Society meeting and suggesting the manipulation used in this experiment.

(Manuscript received February 24, 2004; revision accepted for publication October 7, 2004.) 